International Journal of Pure and Applied Mathematics

Volume 106 No. 2 2016, 355-364

ISSN: 1311-8080 (printed version); ISSN: 1314-3395 (on-line version)

url: http://www.ijpam.eu

doi: 10.12732/ijpam.v106i2.1

\title{
A THEORY OF FUZZY SETS BASED ON CUT SET WITH PARAMETERS
}

\author{
Wang Fengxia ${ }^{1,2} \S$, Zhang Cheng $^{2}$, Yuan Xuehai ${ }^{3}$, Xia Zunquan ${ }^{1}$ \\ ${ }^{1}$ School of Mathematical Sciences \\ Dalian University of Technology \\ Dalian, 116024, P.R. CHINA \\ ${ }^{2}$ School of Information and Engineering \\ Dalian University \\ Dalian, 116622, P.R. CHINA \\ ${ }^{3}$ School of Control Sciences and Engineering \\ Dalian University of Technology \\ Dalian, 116024, P.R. CHINA
}

\begin{abstract}
In this paper, we first discuss the relationships among the existing four cut sets, and introduce the unification of these four cut sets as one cut set with parameters. Then, we give out the properties of the cut set with parameters, and establish the appropriate decomposition theorem and representation theorem of fuzzy sets.
\end{abstract}

AMS Subject Classification: $03 \mathrm{E} 72$

Key Words: fuzzy set, cut set, cut product, cut set with parameters, decomposition theorem, representation theorem

\section{Introduction}

Since the concept of fuzzy set was first introduced by Zadeh (see [1]), many discussions about fuzzy set are proposed. Based on the definitions of cut set

Received: January 29, 2015

Published: February 9, 2016

$\S_{\text {Correspondence author }}$ (c) 2016 Academic Publications, Ltd.

url: www.acadpubl.eu 
and strong cut set of fuzzy set (see [2]), the cut sets, decomposition theorem, and representation theorem are all play very important roles in fuzzy reasoning (see [3] and [4]), fuzzy algebra (see [5] and [6]), fuzzy topology (see [7]), and fuzzy measure, see [8] [9] and [10]. In paper [11], the authors introduced three new cut sets of the fuzzy sets and had obtained four new decomposition theorems and four new representation theorems. And then, in paper [12], four kinds of cut sets and eight mappings on intuitionistic fuzzy sets are introduced, based on which four decomposition theorems and four representation theorems on intuitionistic fuzzy sets are obtained.

Based on paper [12], the equivalence of the four decomposition theorems based on different cut sets for intuitionistic fuzzy sets is proved in the paper [13], as well as the equivalence of the four representation theorems. In fact, the equivalence of the four decomposition theorems and that of four new representation theorems based on different cut sets for fuzzy sets in paper [11] can be proved similarly.

This paper contains three major parts. It first discusses the relationships among the four cut sets in paper [11], then introduces three new cut products and gives the relationships among the cut products in Section 2. In section 3, we introduce the unification of these four cut sets as one cut set with parameters, and give out the properties of the cut set with parameters. Then, in section 4 and section 5 , by using the cut set with parameters, we establish the appropriate decomposition theorem and representation theorem of fuzzy sets.

\section{Preliminary}

Let $X$ be a set, and $A$ is a mapping on $[0,1] \subset R$, that is $A: X \rightarrow[0,1]$, $x \mapsto A(x)$, we call that $A$ is a fuzzy set on $X$, see [1]. In this paper, the class of all fuzzy sets on $X$ is denoted as $\mathcal{F}(X) . \mathcal{P}(X)$ denotes the power set of $X, \lambda \in$ $I=[0,1]$. The following are the definitions of four cut $\operatorname{sets} A_{\lambda}, A^{\lambda}, A_{[\lambda]}, A^{[\lambda]}$ and four strong cut $\operatorname{sets} A_{\underline{\lambda}}, A^{\lambda}, A_{\underline{[\lambda]}}, A \underline{[\lambda]}$ in paper [2] and [11]:

Definition 1.1. Let $A \in \mathcal{F}(X), \lambda \in I$, then:

(1) $A_{\lambda}=\{x \mid x \in X, A(x) \geq \lambda\}$, and $A_{\underline{\lambda}}=\{x \mid x \in X, A(x)>\lambda\}$ are called $\lambda$-upper cut set and $\lambda$-strong upper cut set of fuzzy set $A$ respectively.

(2) $A^{\lambda}=\{x \mid x \in X, A(x) \leq \lambda\}$, and $A^{\lambda}=\{x \mid x \in X, A(x)<\lambda\}$ are called $\lambda$-lower cut set and $\lambda$-strong lower cut set of fuzzy set $A$ respectively. 
(3) $A_{[\lambda]}=\{x \mid x \in X, \lambda+A(x) \geq 1\}$, and $A_{[\lambda]}=\{x \mid x \in X, \lambda+A(x)>1\}$ are called $\lambda$-lower $Q$-cut set and $\lambda$-strong lower $Q$-cut set of fuzzy set $A$ respectively.

(4) $A^{[\lambda]}=\{x \mid x \in X, \lambda+A(x) \leq 1\}$, and $A \underline{[\lambda]}=\{x \mid x \in X, \lambda+A(x)<$ $1\}$ are called $\lambda$-upper $Q$-cut set and $\lambda$-strong upper $Q$-cut set of fuzzy set $A$ respectively.

\section{Relationships Among Four Cut Sets and that of Four Cut Products}

Let $X$ be a set, $A \in \mathcal{F}(X), \lambda \in I=[0,1]$, and $\lambda^{c}=1-\lambda$. By the Definition 1.1 , it is not difficult to prove the relationships among these cut sets as follows:

Property 2.1. (1) $A_{\lambda}=\left(A^{c}\right)^{\lambda^{c}}=A_{\left[\lambda^{c}\right]}=\left(A^{c}\right)^{[\lambda]}$;

(2) $A_{\underline{\lambda}}=\left(A^{c}\right) \underline{\lambda^{c}}=A_{\underline{\left[\lambda^{c}\right]}}=\left(A^{c}\right) \underline{[\lambda]}$;

(3) $A^{\lambda}=\left(A^{c}\right)_{[\lambda]}=A^{\left[\lambda^{c}\right]}=\left(A^{c}\right)_{\lambda^{c}}$;

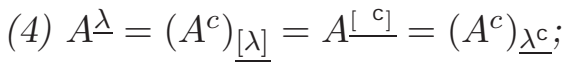

(5) $A_{\lambda}=\left(A^{\underline{\lambda}}\right)^{c}, A^{\lambda}=\left(A_{\underline{\lambda}}\right)^{c}, A_{[\lambda]}=\left(A^{[\lambda]}\right)^{c}, A^{[\lambda]}=\left(A_{\underline{[\lambda]}}\right)^{c}$;

(6) $A_{\lambda}=\left(A^{\left[\lambda^{c}\right]}\right)^{c}, A_{\underline{\lambda}}=\left(A^{\left[\lambda^{c}\right]}\right)^{c}, A^{\lambda}=\left(A_{\underline{\left[\lambda^{c}\right]}}\right)^{c}, A^{\underline{\lambda}}=\left(A_{\left[\lambda^{c}\right]}\right)^{c}$.

The definition of cut product $\lambda A$ we have known is that $\lambda A \in \mathcal{F}(X)$, and $(\lambda A)(x)=\lambda \wedge A(x)$, where $A \in \mathcal{F}(X), \lambda \in I^{[2]}$. In particularly, if $A \in \mathcal{P}(X)$, then $(\lambda A)(x)=\left\{\begin{array}{ll}\lambda, & x \in A \\ 0, & x \notin A\end{array}\right.$, it is the fuzzy subset $\lambda B$ defined in [11]. Similarly, thinking about the other three fuzzy subsets $\lambda \cdot B, \lambda \circ B$, $\lambda \diamond B$ in [11], where $(\lambda \cdot B)(x)=\left\{\begin{array}{l}\lambda, x \in B \\ 1, x \notin B\end{array},(\lambda \circ B)(x)=\left\{\begin{array}{l}1, x \in B \\ \lambda, x \notin B\end{array}\right.\right.$, and $(\lambda \diamond B)(x)=\left\{\begin{array}{c}0, x \in B \\ \lambda, x \notin B\end{array}\right.$, we can define three new cut products:

Definition 2.1. Let $A \in \mathcal{F}(X), \lambda \in I$, we define:

$\lambda \cdot A \in \mathcal{F}(X)$, and $(\lambda \cdot A)(x)=\lambda \vee A^{c}(x) ;$

$\lambda \circ A \in \mathcal{F}(X), \operatorname{and}(\lambda \circ A)(x)=\lambda \vee A(x) ;$ 
$\lambda \diamond A \in \mathcal{F}(X)$, and $(\lambda \diamond A)(x)=\lambda \wedge A^{c}(x)$.

We call the $\lambda \cdot A, \lambda \circ A$ and $\lambda \diamond A$ cut products exactly as $\lambda A$. Then, the following property is clear.

Property 2.2. (1) $\lambda A=\left(\lambda^{c} \cdot A\right)^{c}, \lambda \cdot A=\lambda \circ A^{c}, \lambda \circ A=\left(\lambda^{c} \diamond A\right)^{c}, \lambda \diamond A=\lambda A^{c}$;

(2) $(\lambda A)^{c}=\lambda^{c} \circ A^{c},(\lambda \circ A)^{c}=\lambda^{c} A^{c},(\lambda \cdot A)^{c}=\lambda^{c} \diamond A^{c},(\lambda \diamond A)^{c}=\lambda^{c} \cdot A^{c}$.

Proof. We only prove $\lambda A=\left(\lambda^{c} \cdot A\right)^{c}$, and the others can be proved by the same method. For any $x \in X, \lambda \in I$, we have $\left(\lambda^{c} \cdot A\right)(x)=\lambda^{c} \vee A^{c}(x)=$ $(1-\lambda) \vee(1-A(x))=1-(\lambda \wedge A(x))=1-(\lambda A)(x)$. That is $\left(\lambda^{c} \cdot A\right)=(\lambda A)^{c}$, it follows that $\lambda A=\left(\lambda^{c} \cdot A\right)^{c}$.

By the Property 2.1 and 2.2, the equivalence of the four decomposition theorems and that of four new representation theorems based on different cut sets for fuzzy sets in paper [11] can be easily proved using the similar method as in paper [13].

\section{A Cut Set with Parameters}

Considering the relationships among the four cut sets provided in Property 2.1 , we find the four cut sets can be unified as one cut set with parameters as following.

Definition 3.1. Let $X$ be a set, $A \in \mathcal{F}(X), \lambda \in[0,1], \alpha, \beta \in[0,1]$, and $f:[0,1] \times \mathcal{F}(X) \rightarrow \mathcal{P}(X)$ is a mapping. We call that $f^{(\alpha, \beta)}(\lambda, A)=\{x \in$ $X \mid(1-\beta) A(x)+\beta(1-A(x)) \geq(1-\alpha) \lambda+\alpha(1-\lambda)\}$ is a $\lambda$-cut set with parameters of fuzzy set $A$.

Moreover,

$$
f^{(\alpha, \beta)}(\underline{\lambda}, A)=\{x \in X \mid(1-\beta) A(x)+\beta(1-A(x))>(1-\alpha) \lambda+\alpha(1-\lambda)\}
$$

is the $\lambda$-strong cut set with parameters of fuzzy set $A$.

Since

$$
\begin{aligned}
f^{(\alpha, \beta)}(\lambda, A)= & \{x \in X \mid(1-\beta) A(x)+\beta(1-A(x)) \geq(1-\alpha) \lambda+\alpha(1-\lambda)\} \\
& =\{x \in X \mid(1-2 \beta) A(x) \geq(1-2 \alpha) \lambda+\alpha-\beta\}
\end{aligned}
$$




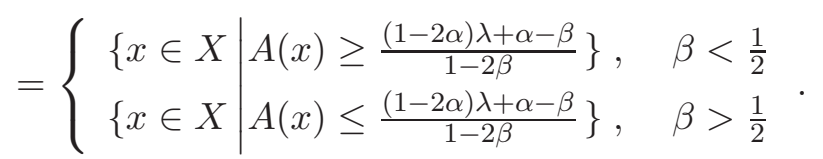

Then, when $\alpha=\beta<\frac{1}{2}$,

$$
f^{(\alpha, \beta)}(\lambda, A)=\{x \in X \mid A(x) \geq \lambda\}=A_{\lambda} ;
$$

when $\alpha=\beta>\frac{1}{2}$,

$$
f^{(\alpha, \beta)}(\lambda, A)=\{x \in X \mid A(x) \leq \lambda\}=A^{\lambda} ;
$$

when $\alpha+\beta=1, \alpha>\frac{1}{2}, \beta<\frac{1}{2}$,

$$
f^{(\alpha, \beta)}(\lambda, A)=\{x \in X \mid A(x) \geq 1-\lambda\}=A_{[\lambda]} ;
$$

when $\alpha+\beta=1, \alpha<\frac{1}{2}, \beta>\frac{1}{2}$,

$$
f^{(\alpha, \beta)}(\lambda, A)=\{x \in X \mid A(x) \leq 1-\lambda\}=A^{[\lambda]} .
$$

In particular, when $\alpha=\beta=0$,

$$
f^{(0,0)}(\lambda, A)=\{x \in X \mid A(x) \geq \lambda\}=A_{\lambda} ;
$$

when $\alpha=\beta=1$,

$$
f^{(1,1)}(\lambda, A)=\{x \in X \mid A(x) \leq \lambda\}=A^{\lambda}
$$

when $\alpha=1, \beta=0$,

$$
f^{(1,0)}(\lambda, A)=\{x \in X \mid A(x) \geq 1-\lambda\}=A_{[\lambda]} ;
$$

when $\alpha=0, \beta=1$,

$$
f^{(0,1)}(\lambda, A)=\{x \in X \mid A(x) \leq 1-\lambda\}=A^{[\lambda]} .
$$

So, the $\lambda$-cut set with parameters is a promotion form of the four cut sets in paper [11]. That is also can be said that the $\lambda$-cut set with parameters is a unification form of the four cut sets in paper [11].

Let $A^{t}, A, B \in \mathcal{F}(X)=\{C \mid C: X \rightarrow[0,1]$ is a mapping $\}, \lambda, \lambda_{1}, \lambda_{2}, \alpha_{t} \in$ $I=[0,1], t \in T$, and $a=\vee_{t \in T} \alpha_{t}, b=\wedge_{t \in T} \alpha_{t}$. We have the following properties of the $\lambda$-cut set with parameters. 
Property 3.1. (1) When $\beta<\frac{1}{2}$,

$$
\begin{aligned}
& f^{(\alpha, \beta)}(\lambda, A \cup B)=f^{(\alpha, \beta)}(\lambda, A) \cup f^{(\alpha, \beta)}(\lambda, B), \\
& f^{(\alpha, \beta)}(\underline{\lambda}, A \cup B)=f^{(\alpha, \beta)}(\underline{\lambda}, A) \cup f^{(\alpha, \beta)}(\underline{\lambda}, B), \\
& f^{(\alpha, \beta)}(\lambda, A \cap B)=f^{(\alpha, \beta)}(\lambda, A) \cap f^{(\alpha, \beta)}(\lambda, B), \\
& f^{(\alpha, \beta)}(\underline{\lambda}, A \cap B)=f^{(\alpha, \beta)}(\underline{\lambda}, A) \cap f^{(\alpha, \beta)}(\underline{\lambda}, B) .
\end{aligned}
$$

If $\beta>\frac{1}{2}$, then

$$
\begin{aligned}
& f^{(\alpha, \beta)}(\lambda, A \cup B)=f^{(\alpha, \beta)}(\lambda, A) \cap f^{(\alpha, \beta)}(\lambda, B), \\
& f^{(\alpha, \beta)}(\underline{\lambda}, A \cup B)=f^{(\alpha, \beta)}(\underline{\lambda}, A) \cap f^{(\alpha, \beta)}(\underline{\lambda}, B), \\
& f^{(\alpha, \beta)}(\lambda, A \cap B)=f^{(\alpha, \beta)}(\lambda, A) \cup f^{(\alpha, \beta)}(\lambda, B), \\
& f^{(\alpha, \beta)}(\underline{\lambda}, A \cap B)=f^{(\alpha, \beta)}(\underline{\lambda}, A) \cup f^{(\alpha, \beta)}(\underline{\lambda}, B) .
\end{aligned}
$$

(2) If $\alpha<\frac{1}{2}$, then

$$
\begin{gathered}
\lambda_{1}<\lambda_{2} \Rightarrow f^{(\alpha, \beta)}\left(\lambda_{1}, A\right) \supseteq f^{(\alpha, \beta)}\left(\lambda_{2}, A\right), \quad f^{(\alpha, \beta)}\left(\underline{\lambda_{1}}, A\right) \supseteq f^{(\alpha, \beta)}\left(\underline{\lambda_{2}}, A\right), \\
f^{(\alpha, \beta)}\left(\lambda_{1}, A\right) \supseteq f^{(\alpha, \beta)}\left(\underline{\lambda_{1}}, A\right), \quad f^{(\alpha, \beta)}\left(\underline{\lambda_{1}}, A\right) \supseteq f^{(\alpha, \beta)}\left(\lambda_{2}, A\right) .
\end{gathered}
$$

If $\alpha>\frac{1}{2}$, then

$$
\begin{gathered}
\lambda_{1}<\lambda_{2} \Rightarrow f^{(\alpha, \beta)}\left(\lambda_{1}, A\right) \subseteq f^{(\alpha, \beta)}\left(\lambda_{2}, A\right), \quad f^{(\alpha, \beta)}\left(\underline{\lambda_{1}}, A\right) \subseteq f^{(\alpha, \beta)}\left(\underline{\lambda_{2}}, A\right), \\
f^{(\alpha, \beta)}\left(\lambda_{1}, A\right) \supseteq f^{(\alpha, \beta)}\left(\underline{\lambda_{1}}, A\right), \quad f^{(\alpha, \beta)}\left(\lambda_{1}, A\right) \subseteq f^{(\alpha, \beta)}\left(\underline{\lambda_{2}}, A\right) .
\end{gathered}
$$

(3) If $\beta<\frac{1}{2}$, then

$$
\begin{aligned}
& f^{(\alpha, \beta)}\left(\lambda, \bigcup_{t \in T} A^{t}\right) \supseteq \bigcup_{t \in T} f^{(\alpha, \beta)}\left(\lambda, A^{t}\right), \\
& f^{(\alpha, \beta)}\left(\underline{\lambda}, \bigcup_{t \in T} A^{t}\right)=\bigcup_{t \in T} f^{(\alpha, \beta)}\left(\underline{\lambda}, A^{t}\right), \\
& f^{(\alpha, \beta)}\left(\lambda, \bigcap_{t \in T} A^{t}\right)=\bigcap_{t \in T} f^{(\alpha, \beta)}\left(\lambda, A^{t}\right), \\
& f^{(\alpha, \beta)}\left(\underline{\lambda}, \bigcap_{t \in T} A^{t}\right)=\bigcap_{t \in T} f^{(\alpha, \beta)}\left(\underline{\lambda}, A^{t}\right) .
\end{aligned}
$$

If $\beta>\frac{1}{2}$, then

$$
f^{(\alpha, \beta)}\left(\lambda, \bigcup_{t \in T} A^{t}\right)=\bigcap_{t \in T} f^{(\alpha, \beta)}\left(\lambda, A^{t}\right),
$$




$$
\begin{aligned}
& f^{(\alpha, \beta)}\left(\underline{\lambda}, \bigcup_{t \in T} A^{t}\right) \subseteq \bigcap_{t \in T} f^{(\alpha, \beta)}\left(\underline{\lambda}, A^{t}\right), \\
& f^{(\alpha, \beta)}\left(\lambda, \bigcap_{t \in T} A^{t}\right) \supseteq \bigcup_{t \in T} f^{(\alpha, \beta)}\left(\lambda, A^{t}\right), \\
& f^{(\alpha, \beta)}\left(\underline{\lambda}, \bigcap_{t \in T} A^{t}\right) \subseteq \bigcup_{t \in T} f^{(\alpha, \beta)}\left(\underline{\lambda}, A^{t}\right) .
\end{aligned}
$$

(4) $f^{(\alpha, \beta)}\left(\lambda, A^{c}\right)=\left(f^{(\alpha, \beta)}\left(\underline{\lambda^{c}}, A\right)\right)^{c}, f^{(\alpha, \beta)}\left(\underline{\lambda}, A^{c}\right)=\left(f^{(\alpha, \beta)}\left(\lambda^{c}, A\right)\right)^{c}$.

(5) If $\alpha<\frac{1}{2}$, then

$$
\begin{aligned}
& f^{(\alpha, \beta)}(a, A)=\bigcap_{t \in T} f^{(\alpha, \beta)}\left(\alpha_{t}, A\right), \\
& f^{(\alpha, \beta)}(b, A) \supseteq \bigcup_{t \in T} f^{(\alpha, \beta)}\left(\alpha_{t}, A\right), \\
& f^{(\alpha, \beta)}(\underline{a}, A) \subseteq \bigcap_{t \in T} f^{(\alpha, \beta)}\left(\underline{\alpha_{t}}, A\right), \\
& f^{(\alpha, \beta)}(\underline{b}, A)=\bigcup_{t \in T} f^{(\alpha, \beta)}\left(\underline{\alpha_{t}}, A\right) .
\end{aligned}
$$

If $\alpha>\frac{1}{2}$, then

$$
\begin{aligned}
& f^{(\alpha, \beta)}(a, A) \supseteq \bigcup_{t \in T} f^{(\alpha, \beta)}\left(\alpha_{t}, A\right), \\
& f^{(\alpha, \beta)}(b, A)=\bigcap_{t \in T} f^{(\alpha, \beta)}\left(\alpha_{t}, A\right), \\
& f^{(\alpha, \beta)}(\underline{a}, A)=\bigcup_{t \in T} f^{(\alpha, \beta)}\left(\underline{\alpha_{t}}, A\right), \\
& f^{(\alpha, \beta)}(\underline{b}, A) \subseteq \bigcap_{t \in T} f^{(\alpha, \beta)}\left(\underline{\alpha_{t}}, A\right) .
\end{aligned}
$$

\section{The Decomposition Theorems Based on the Cut Set with}

\section{Parameters}

Let $\phi_{i}:[0,1] \times \mathcal{P}(X) \rightarrow L^{X},(\lambda, A) \mapsto \phi_{i}(\lambda, A)$ are some mappings $(i=$ $1,2, \ldots, 8)$, where

$$
\begin{gathered}
\phi_{1}(\lambda, A)(x)=\left\{\begin{array}{ll}
0, & A(x)=0, \\
\lambda, & A(x)=1 .
\end{array} \quad \phi_{2}(\lambda, A)(x)= \begin{cases}\lambda, & A(x)=0, \\
1, & A(x)=1 .\end{cases} \right. \\
\phi_{3}(\lambda, A)(x)=\left\{\begin{array}{ll}
1-\lambda, & A(x)=0, \\
0, & A(x)=1 .
\end{array} \quad \phi_{4}(\lambda, A)(x)= \begin{cases}1, & A(x)=0, \\
1-\lambda, & A(x)=1 .\end{cases} \right.
\end{gathered}
$$




$$
\begin{aligned}
& \phi_{5}(\lambda, A)(x)=\left\{\begin{array}{lc}
0, & A(x)=0, \\
1-\lambda, & A(x)=1 .
\end{array} \quad \phi_{6}(\lambda, A)(x)= \begin{cases}1-\lambda, & A(x)=0, \\
1, & A(x)=1 .\end{cases} \right. \\
& \phi_{7}(\lambda, A)(x)=\left\{\begin{array}{ll}
\lambda, & A(x)=0, \\
0, & A(x)=1 .
\end{array} \quad \phi_{8}(\lambda, A)(x)= \begin{cases}1, & A(x)=0, \\
\lambda, & A(x)=1 .\end{cases} \right.
\end{aligned}
$$

Theorem 4.1. When $i=1,7, j=2,8, \alpha=\beta$,

(1) $A=\bigcup_{\lambda \in[0,1]} \phi_{i}\left(\lambda, f^{(\alpha, \beta)}(\lambda, A)\right)=\bigcap_{\lambda \in[0,1]} \phi_{j}\left(\lambda, f^{(\alpha, \beta)}(\lambda, A)\right)$.

(2) $A=\bigcup_{\lambda \in[0,1]} \phi_{i}\left(\lambda, f^{(\alpha, \beta)}(\underline{\lambda}, A)\right)=\bigcap_{\lambda \in[0,1]} \phi_{j}\left(\lambda, f^{(\alpha, \beta)}(\underline{\lambda}, A)\right)$.

(3) Let the mapping $H: I \rightarrow \mathcal{P}(X), \lambda \mapsto H(\lambda)$ satisfied the condition $f^{(\alpha, \beta)}(\underline{\lambda}, A) \subseteq H(\lambda) \subseteq f^{(\alpha, \beta)}(\lambda, A), \forall \lambda \in I$, then

(i) $A=\bigcup_{\lambda \in[0,1]} \phi_{i}(\lambda, H(\lambda))=\bigcap_{\lambda \in[0,1]} \phi_{j}(\lambda, H(\lambda))$.

(ii) $\lambda_{1}<\lambda_{2} \Rightarrow H\left(\lambda_{1}\right) \supseteq H\left(\lambda_{2}\right)\left(\alpha=\beta<\frac{1}{2}\right)$,

$\lambda_{1}<\lambda_{2} \Rightarrow H\left(\lambda_{1}\right) \subseteq H\left(\lambda_{2}\right)\left(\alpha=\beta>\frac{1}{2}\right)$.

(iii) $f^{(\alpha, \beta)}(\lambda, A)=\bigcap_{\alpha<\lambda} H(\alpha), f^{(\alpha, \beta)}(\underline{\lambda}, A)=\bigcup_{\alpha>\lambda} H(\alpha)\left(\alpha=\beta<\frac{1}{2}\right)$,

$f^{(\alpha, \beta)}(\lambda, A)=\bigcap_{\alpha>\lambda} H(\alpha), f^{(\alpha, \beta)}(\underline{\lambda}, A)=\bigcup_{\alpha<\lambda} H(\alpha)\left(\alpha=\beta>\frac{1}{2}\right)$.

Here $\bigcup_{t \in \Phi} A^{(t)}=\Phi, \bigcap_{t \in \Phi} A^{(t)}=X$.

Theorem 4.2. When $i=3,5, j=4,6, \alpha+\beta=1$,

(1) $A=\bigcup_{\lambda \in[0,1]} \phi_{i}\left(\lambda, f^{(\alpha, \beta)}(\lambda, A)\right)=\bigcap_{\lambda \in[0,1]} \phi_{j}\left(\lambda, f^{(\alpha, \beta)}(\lambda, A)\right)$.

(2) $A=\bigcup_{\lambda \in[0,1]} \phi_{i}\left(\lambda, f^{(\alpha, \beta)}(\underline{\lambda}, A)\right)=\bigcap_{\lambda \in[0,1]} \phi_{j}\left(\lambda, f^{(\alpha, \beta)}(\underline{\lambda}, A)\right)$.

(3) Let the mapping $H: I \rightarrow \mathcal{P}(X), \lambda \mapsto H(\lambda)$ satisfied the condition $f^{(\alpha, \beta)}(\underline{\lambda}, A) \subseteq H(\lambda) \subseteq f^{(\alpha, \beta)}(\lambda, A), \forall \lambda \in I$, then

(i) $A=\bigcup_{\lambda \in[0,1]} \phi_{i}(\lambda, H(\lambda))=\bigcap_{\lambda \in[0,1]} \phi_{j}(\lambda, H(\lambda))$.

(ii) $\lambda_{1}<\lambda_{2} \Rightarrow H\left(\lambda_{1}\right) \supseteq H\left(\lambda_{2}\right)\left(\alpha<\frac{1}{2}, \beta>\frac{1}{2}\right)$,

$\lambda_{1}<\lambda_{2} \Rightarrow H\left(\lambda_{1}\right) \subseteq H\left(\lambda_{2}\right)\left(\alpha>\frac{1}{2}, \beta<\frac{1}{2}\right)$.

(iii) $f^{(\alpha, \beta)}(\lambda, A)=\bigcap_{\alpha<\lambda} H(\alpha), f^{(\alpha, \beta)}(\underline{\lambda}, A)=\bigcup_{\alpha>\lambda} H(\alpha)\left(\alpha<\frac{1}{2}, \beta>\frac{1}{2}\right)$,

$f^{(\alpha, \beta)}(\lambda, A)=\bigcap_{\alpha>\lambda} H(\alpha), f^{(\alpha, \beta)}(\underline{\lambda}, A)=\bigcup_{\alpha<\lambda} H(\alpha)\left(\alpha>\frac{1}{2}, \beta<\frac{1}{2}\right)$. 
Here $\bigcup_{t \in \Phi} A^{(t)}=\Phi, \bigcap_{t \in \Phi} A^{(t)}=X$.

It is easy to prove that the four decomposition theorems in paper [11] are particular cases of the above two theorems.

\section{The Representation Theorems Based on the Cut Set With Parameters}

Let $\mathcal{U}(X)$ be a set of all set embedding over $X, H \in \mathcal{U}(X), T_{i}: \mathcal{U}(X) \rightarrow$ $\mathcal{F}(X), H \rightarrow T_{i}(H)(i=1,2,3,4)$, where

$$
\begin{aligned}
& T_{1}(H)=\bigcup_{\lambda \in[0,1]} \phi_{1}(\lambda, H(\lambda)), \quad T_{2}(H)=\bigcap_{\lambda \in[0,1]} \phi_{2}(\lambda, H(\lambda)), \\
& T_{3}(H)=\bigcap_{\lambda \in[0,1]} \phi_{4}(\lambda, H(\lambda)), \quad T_{4}(H)=\bigcup_{\lambda \in[0,1]} \phi_{3}(\lambda, H(\lambda)) .
\end{aligned}
$$

Let $\mathcal{V}(X)$ be a set of all order set embedding over $X, H \in \mathcal{V}(X), T_{i}: \mathcal{V}(X) \rightarrow$ $\mathcal{F}(X), H \rightarrow T_{i}(H)(i=5,6,7,8)$, where

$$
\begin{aligned}
& T_{5}(H)=\bigcap_{\lambda \in[0,1]} \phi_{8}(\lambda, H(\lambda)), \quad T_{6}(H)=\bigcup_{\lambda \in[0,1]} \phi_{7}(\lambda, H(\lambda)), \\
& T_{7}(H)=\bigcup_{\lambda \in[0,1]} \phi_{5}(\lambda, H(\lambda)), \quad T_{8}(H)=\bigcap_{\lambda \in[0,1]} \phi_{6}(\lambda, H(\lambda)) .
\end{aligned}
$$

Theorem 5.1. For $T_{i}(i=1,2,5,6)$, we have

(1) When $\alpha=\beta, f^{(\alpha, \beta)}\left(\underline{\eta}, T_{i}(H)\right) \subseteq H(\eta) \subseteq f^{(\alpha, \beta)}\left(\eta, T_{i}(H)\right), \forall \eta \in I$;

(2) $T_{i}\left(\bigcup_{\gamma \in \Gamma} H_{\gamma}\right)=\bigcup_{\gamma \in \Gamma} T_{i}\left(H_{\gamma}\right), T_{i}\left(\bigcap_{\gamma \in \Gamma} H_{\gamma}\right)=\bigcap_{\gamma \in \Gamma} T_{i}\left(H_{\gamma}\right), T_{i}\left(H^{c}\right)=\left(T_{i}(H)\right)^{c}$.

Theorem 5.2. For $T_{i}(i=3,4,7,8)$, we have

(1) When $\alpha+\beta=1, f^{(\alpha, \beta)}\left(\underline{\eta}, T_{i}(H)\right) \subseteq H(\eta) \subseteq f^{(\alpha, \beta)}\left(\eta, T_{i}(H)\right), \forall \eta \in I$;

(2) $T_{i}\left(\bigcup_{\gamma \in \Gamma} H_{\gamma}\right)=\bigcap_{\gamma \in \Gamma} T_{i}\left(H_{\gamma}\right), T_{i}\left(\bigcap_{\gamma \in \Gamma} H_{\gamma}\right)=\bigcup_{\gamma \in \Gamma} T_{i}\left(H_{\gamma}\right), T_{i}\left(H^{c}\right)=\left(T_{i}(H)\right)^{c}$.

It is also easy to prove that the four representation theorems in paper [11] are particular cases of the above two theorems. 


\section{Conclusions}

In this paper, a cut set with parameters is presented as the unification of the existing four cut sets and its properties are discussed. Based on the cut set with parameters, the appropriate decomposition theorem and representation theorem of fuzzy sets are established. These discussions extended the theories of fuzzy sets.

\section{References}

[1] L. A. Zadeh, Fuzzy sets, Information and Control, 8(3) (1965), 338-353. DOI: 10.1016/S0019-9958(65)90241-X.

[2] D. Dubois, H. Prade, Fuzzy Sets and Systems, Academic Press, New York (1980). ISBN13: 9780122227509 .

[3] C.Z. Luo, Z.P. Wang, Representation of fuzzy reasoning, Fuzzy Sets and Systems, 36 (1990), 77-81. DOI: 10.1016/S0165-0114(90)90080-p.

[4] D. Dubois, E. Hullermeier, H. Prade, On the representation of fuzzy rules in terms of crisp rules, Information Sciences, 151 (2003), 301-326. DOI: 10.1016/S0020-0255(02)00403-6.

[5] J.N. Mordeson, D.S. Malik,Fuzzy Commutative Algebra, World Scientific Publishing, Singapore(1998). ISBN13: 9789810236281.

[6] J.N. Mordeson, K.R. Bhutani, A. Rosenfeld, Fuzzy Group Theory, Springer, New York(2005). ISBN13: 9783642064128.

[7] Y.M. Liu, M.K. Luo, Fuzzy Topology, World Scientific Publishing, Singapore(1990). ISBN13: 9789810228620.

[8] C. Bertoluzza, M. Solci, M.L. Caodieci, Measure of a fuzzy set: The $\alpha$-cut approach in the finite case, Fuzzy Sets and Systems, 123(2001), 93-102. DOI:10.1016/S01650114(00)00074-9.

[9] J.N. Garcia, Z. Kutalik, K.H. Cho, O. Wolkenhauer, Level sets and the minimum volume sets of probability density function, International Journal of Approximate Reasoning, 34 (2003), 25-47. DOI: 10.1016/S0888-613X (03)00052-5.

[10] E. Pap, D. Surla, Lebesgue measure of $\alpha$-cuts approach for finding the height of the membership function, Fuzzy Sets and Systems, 111 (2000), 341-350. DOI:10.1016/S01650114(98)00162-6.

[11] X.H. Yuan, H.X. Li, E.S. Lee, Three new cut sets of fuzzy sets and new theories of fuzzy sets, Computers and Mathematics with Applications, 57(2009), 691-701. DOI:10.1016/j.camwa.2008.05.044.

[12] Yuan X H, Li H X, Sun K B, The cut sets, decomposition theorems and representation theorems on intuitionistic fuzzy sets and interval valued fuzzy sets, SCIENCE CHINA (Information Sciences), 54(1) (2011), 91-110. DOI:10.1007/s11432-010-4078-6.

[13] Fengxia Wang, Cheng Zhang, Zunquan Xia, Equivalence of the cut sets-based decomposition theorems and representation theorems on intuitionistic fuzzy sets and interval-valued fuzzy sets, Mathematical and Computer Modelling, 57(2013), 1364-1370. DOI:10.1016/j.mcm.2012.11.025. 